\title{
The Variable Structure of the X-ray Corona as Viewed by Yohkoh
}

\author{
By KAZUNARI SHIBATA
}

Solar Physics Division, National Astronomical Observatory, Mitaka, Tokyo 181, Japan

Recent findings on the variable structure of the corona by the soft X-ray telescope on board Yohkoh are reviewed, with emphasis upon the structure and evolution of active regions, and the long term evolution of the global structure of the corona.

\section{Introduction}

The Solar-A spacecraft (Ogawara et al. 1991) was successfully launched on August 30, 1991 (Ogawara et al. 1992), and was named Yohkoh which means "sunbeam" in Japanese. Yohkoh carries a soft X-ray telescope (SXT) (Tsuneta et al. 1991) that has been producing an unprecedented sequence of whole-Sun images at 3 - $60 \AA$ (Acton et al. 1992). The routine data typically consist of some tens of whole-Sun images (FFI = full frame images; see Figure 1) per day, with much higher time resolution for specific observations of restricted areas (PFI = partial frame images), such as active regions or flares. The X-ray emission reveals the connectivity of the coronal magnetic field, and also shows where strong coronal heating is occurring. In particular, the movie of the whole-Sun SXT images has revealed that the solar corona is much more dynamic than had been thought, i.e., the corona is full of transient loop brightenings (Shimizu et al. 1992), jets (Shibata et al. 1992b), expanding magnetic loops (Uchida et al. 1992), global restructuring of coronal fields (Tsuneta et al. 1992a), etc.

In this article, I shall review recent findings on variable structure of corona by Yohkoh/ SXT, mainly on the following subjects: (1) structure and evolution of active regions (from emerging flux regions to global restructuring events) and (2) long term evolution of global structure of the corona (mainly based on X-ray synoptic charts). A preliminary report on the white light observations by the aspect sensor of Yohkoh/SXT is given by Hudson (1994). The reader should refer to the Yohkoh special issue of Publ. Astr. Soc. Japan (1992, vol. 44, No. 5), Acton et al. (1992), and Tsuneta \& Lemen (1993) for reviews of initial discoveries by Yohkoh/SXT, and to Hudson (1993), Uchida (1993), and Tsuneta (1993) for reviews of flare observations by Yohkoh/SXT. See also Antonucci (1994) for a review of earlier soft X-ray observation.

\section{Structure and evolution of active regions}

Structure and Evolution of EFRs: Emerging flux regions are observed as very bright compact regions in SXT images, and hence are easily recognized in FFIs. They rapidly grow in size and in brightness (Figure 2), with rapid fluctuations in shape and brightness (Kawai et al. 1992), often accompanied by small flares and jets (Shibata et al. 1992b). In general, the SXT bright features coincide well in space with $\mathrm{H} \alpha$ arch filament systems (Kawai et al. 1992). Ishido et al. (1992) studied the size evolution of several EFRs and found that the apparent velocity of the increase in size of EFRs is large $(\sim 3-5 \mathrm{~km} / \mathrm{s})$ in the very early phase (less than 5 hours after birth), but is small $(\sim 1-2 \mathrm{~km} / \mathrm{s})$ in the later phase. This evolution is similar to that of the separation velocity of the two footpoints of the emerging flux (Harvey \& Martin 1973; Chou 1993), although the expansion speed of 
Figure 1. A full Sun soft X-ray image (negative) taken by Yohkoh/SXT on Nov. 12, 1991. The image was taken at an effective energy of $\sim 1 \mathrm{keV}$ with 5 " pixels and $2.67 \mathrm{sec}$ exposure time (a compressed image is shown here). Note that an X-ray jet is ejected from a small active region (NOAA 6918) in the northwest (upper right) quadrant of the solar disk (Shibata et al. 1992b).

coronal X-ray loops is larger than the separation velocity $(<1.2 \mathrm{~km} / \mathrm{s})$. Large expansion speeds of the coronal parts of emerging flux loops has also been found theoretically by Shibata et al. (1989) using 2D MHD numerical simulations, and the model has been extended to 3D by Matsumoto et al. (1993).

A preliminary study of the thermal evolution of an EFR (NOAA7150) by Su et al. (1993) using the filter ratio method (Hara et al. 1992) has shown that the average temperature of the EFR increases with age from $2 \times 10^{6} \mathrm{~K}$ to $3.5 \times 10^{6} \mathrm{~K}$ in 3 days. Considering the very rapid increase in size of EFRs, this seems to be consistent with the well-known scaling law of Rosner et al. (1978), but may not be necessarily in agreement with the analysis of Skylab data by Little and Krieger cited by Webb (1981).

Interaction of EFRs with Pre-existing Magnetic Fields: Shibata et al. (1993a) have 


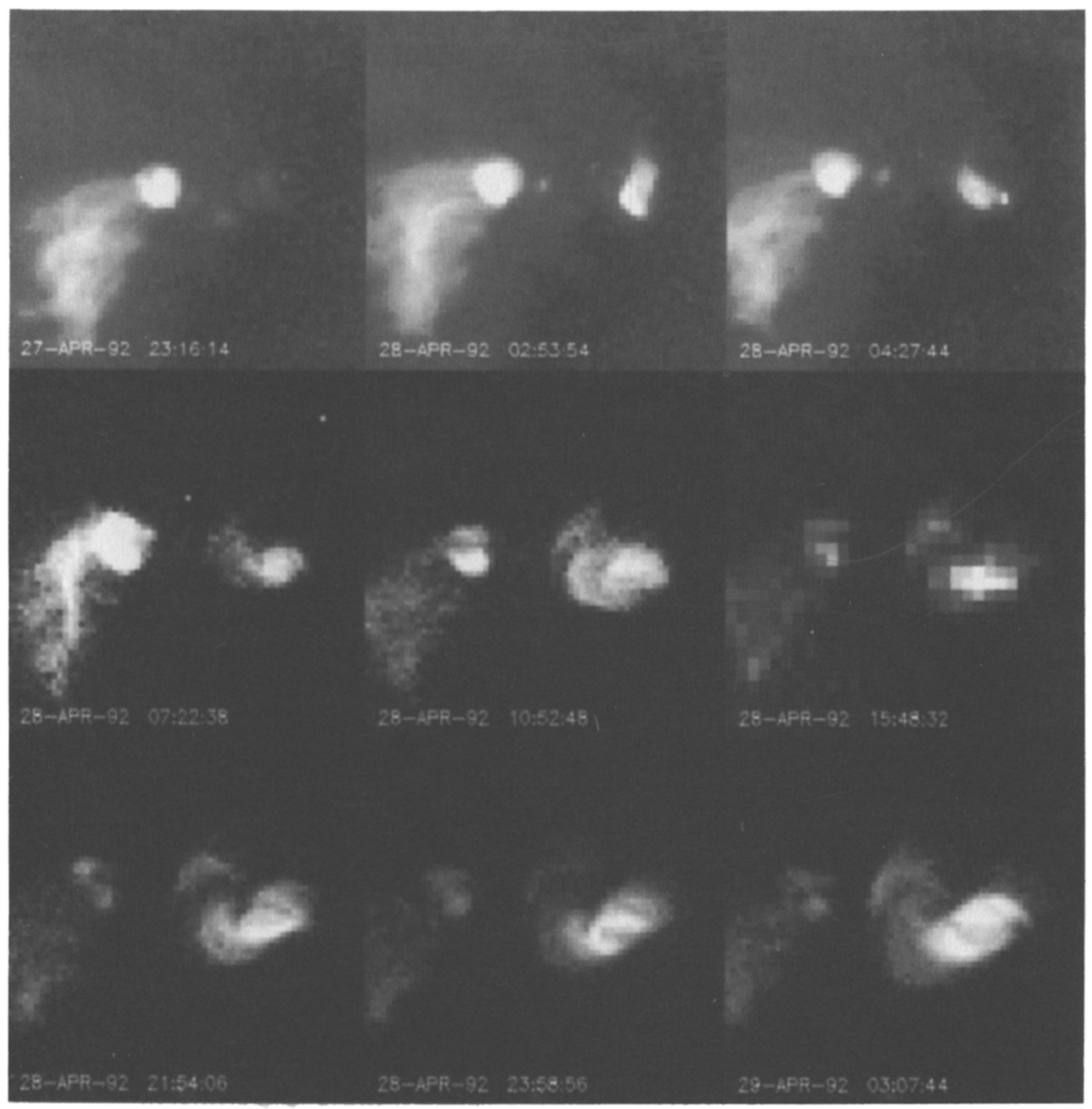

FIGURE 2. The rapid growth of emerging flux region, NOAA7150 (courtesy of G. Kawai). The size of each frame is $640 " \times 640 "$.

studied the interaction between EFRs and overlying coronal magnetic fields, and found that there are fundamentally two types of interaction (reconnection) (see Figure 3):

(1) Anemone-jet type: This type occurs when emerging flux appears in a coronal hole (Figure 4 for a typical example). The EFR (or AR) looks like a "sea anemone", and an $\alpha$-type sunspot is sometimes seen at the center of the "anemone". In many regions, jets (Shibata et al. 1992b) are often ejected from the AR in the vertical direction, which suggests reconnection between emerging flux and the (nearly vertical) coronal fields of the surrounding unipolar region.

(2) Double-loops (Two-sided-loops) type: This type is seen when emerging flux appears in a quiet region. Large-scale loop brightenings occur on both sides of the emerging flux, suggesting reconnection between the emerging flux and an overlying (nearly horizontal) coronal magnetic field. The loop brightenings seem to correspond to the jets occurring in closed loop systems. A reconnection model of two-sided loops has been developed by Yokoyama \& Shibata (1993) by using 2D resistive MHD numerical simulations (Shibata et al. 1992a), extending their previous emerging flux model (Shibata et al. 1989). 


\section{Coronal Hole Quiet Region}

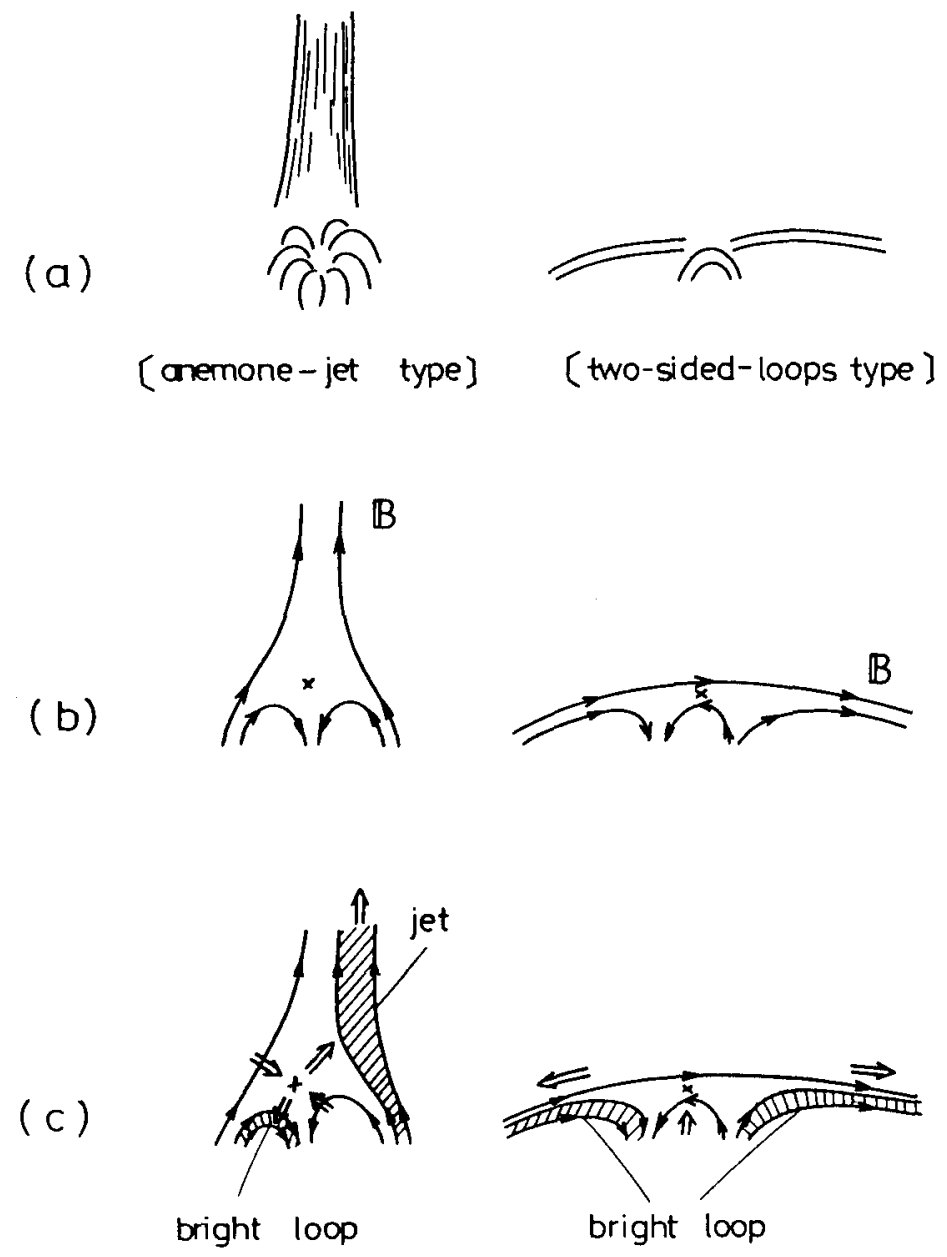

FIGURE 3. Schematic illustration of two types of interaction between emerging flux regions and overlying coronal fields (Shibata et al. 1993a).

Jets: The Yohkoh/SXT has revealed that there are many jet-like features in the solar corona, i.e., transitory $\mathrm{X}$-ray enhancements with an apparent collimated motion (Shibata et al. 1992b; Strong et al. 1992). According to preliminary studies, the length of jets found in FFI ranges from $10^{4} \mathrm{~km}$ to more than $4 \times 10^{5} \mathrm{~km}$, the apparent projection velocity is within a few 10 and $1000 \mathrm{~km} / \mathrm{s}$, though most of jets have velocities less than a few $100 \mathrm{~km} / \mathrm{s}$, and the total kinetic energy of these jets is estimated to be $10^{25}-10^{28} \mathrm{erg}$. Almost all jets except for limb events are associated with flares in X-ray bright points (see Strong et al. 1992; Nitta et al. 1992; Harvey et al. 1993, for study of XBPs found by Yohkoh) emerging flux regions, and active regions. They sometimes occur several times from the same X-ray feature. It is likely that some flare-like loop brightenings (e.g., Shimizu et al. 1992) correspond to jets occurring in closed loop systems. As discussed above, various types of magnetic reconnection especially interactions between emerging 


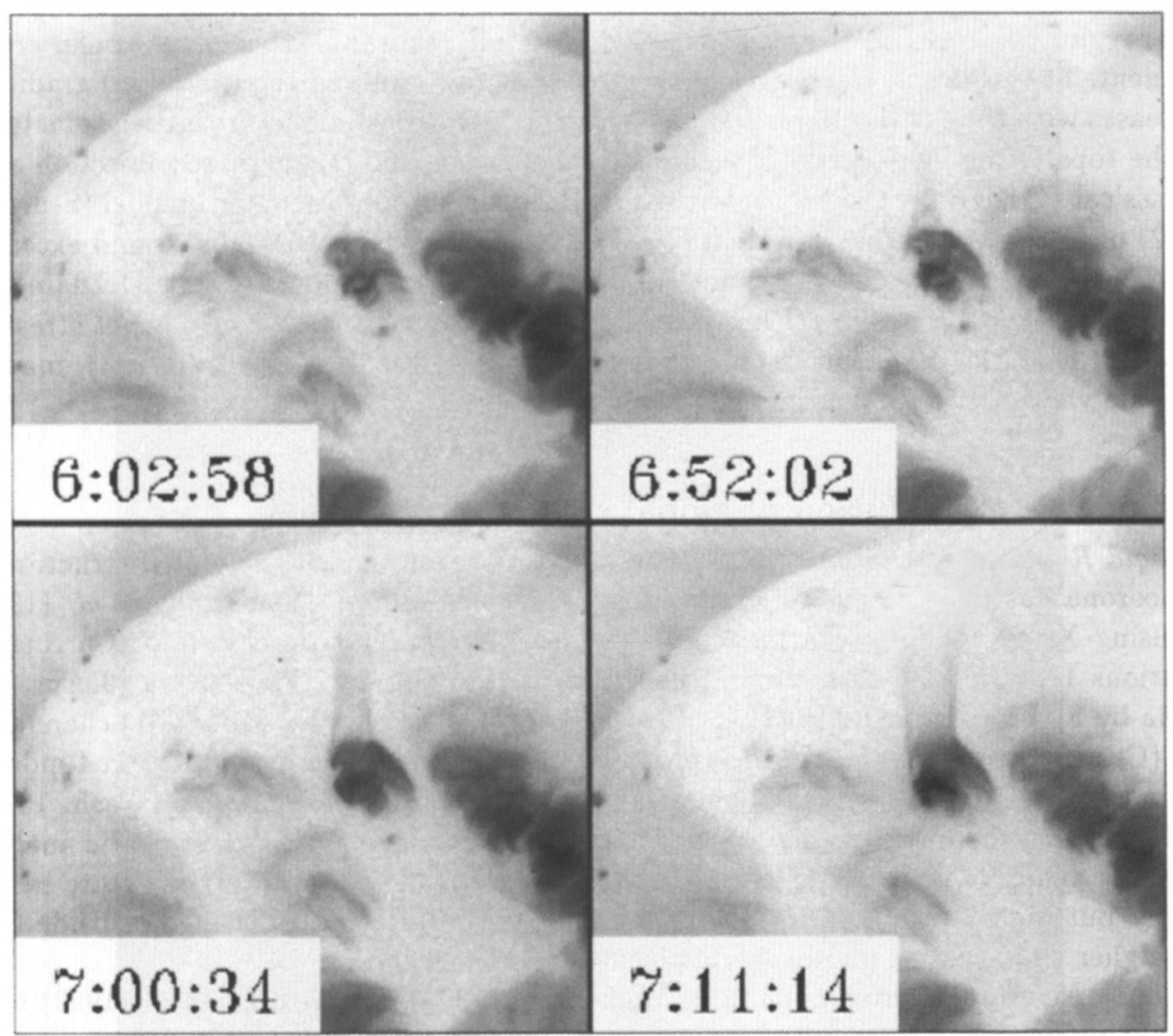

FIgURE 4. A typical example of an "anemone-jet" that occurred in NOAA 7001 on 1992 Jan. 11 (Shibata et al. 1993b). The shape of the active region at the footpoint of the jet looks like sea anemone, and hence this $\mathrm{AR}$ is called anemone-AR. The surrounding region is a coronal hole. The maximum length is probably greater than $3 \times 10^{5} \mathrm{~km}$, and the apparent velocity is within the range of $90-240 \mathrm{~km} / \mathrm{s}$.

flux (or expanding magnetic flux) and pre-existing fields have been suggested to play a central role in generating these X-ray jets (Shibata et al. 1992b).

$A R$ Expansion and $C M E s$ : The SXT movie revealed that almost all active regions show continual or intermittent expansion with a velocity from a few to a few $10 \mathrm{~km} / \mathrm{s}$ (Uchida et al. 1992). (It is interesting to note here that the opposite case also occurs; shrinking loops were clearly observed in an active region NOAA7420 during the week of Feb. $7-14,1993$.) The slower events (at a few $\mathrm{km} / \mathrm{s}$ ) seem to be a result of growth of ARs due to succesive emergence of magnetic flux as discussed above, whereas the faster expansion (at a few $10 \mathrm{~km} / \mathrm{s}$ ) may be physically more similar to a CME (Watanabe et al. 1992; Klimchuk et al. 1993). A typical example of the faster events occurred at the east limb of the Sun on Dec. 9, 1991, as reported by Uchida et al. (1992).

Global Restructuring of Coronal Magnetic Fields Associated with Filament Eruption: It has been found that large arcade structures suddenly (in a time scale of 30 min to 1 day) appear in SXT images after filament eruption (or filament disappearance) (Watanabe et al. 1992; McAllister et al. 1992; Tsuneta et al. 1992a; Hanaoka et al. 1993). The size of 
arcade structure ranges from $10^{5} \mathrm{~km}$ to $\sim 10^{6} \mathrm{~km}$, making them almost global in scope in extreme cases. Such a global restructuring event occurred on Nov. 12, 1991, near the polar region (Tsuneta et al. 1992a) in association with a disappearance of the polar crown filament. In this event, the height of the arcade (a few hundred thousand $\mathrm{km}$ ) gradually increases with time with a velocity of a few $\mathrm{km} / \mathrm{s}$. A cusp-like structure is seen sometimes at the tops of the loops forming such an arcade, suggesting magnetic reconnection as in a classical CSHKP (= Carmichael-Sturrock-Hirayama-Kopp-Pneuman) model (Sturrock 1992) of CME-related flares (Shibata 1991). Note that Yohkoh/SXT has found excellent examples of this class of flares, which show beautiful cusp shapes, e.g., an LDE flare that occurred on Feb. 21, 1992 at the west limb (Tsuneta et al. 1992b). A helmet streamer observed near the pole, such as the event on Jan. 24, 1992, (Hiei et al. 1993), may be the side view of this kind of global restructuring event.

\section{Long term evolution of global structure of corona}

Rigid Rotation of Coronal Holes: The long-term evolution of the global structure of the corona has been extensively studied by Takahashi (1993) and Kozuka et al. (1993) by using X-ray synoptic charts. Figure 5 shows X-ray synoptic charts for Carrington rotations 1851 (from 5 Jan. 92 to 1 Feb. 92) to 1854 (from 27 Mar. 92 to 23 Apr. 92) made by Slater \& Lemen (1993). It is seen that the dark coronal hole (CH) channel at $45^{\circ}$ (Carrington longitude) keeps approximately the same shape for more than 4 months (from Feb. 92 to May 92), i.e., shows rigid rotation (Takahashi 1993, Hayashi 1992). This confirms the discovery by Skylab (Timothy et al. 1975). From systematic analysis of X-ray synoptic charts, Takahashi (1993) found that low-latitude CHs rotate nearly rigidly but high latitude $\mathrm{CHs}$ do not, although the rotation rates of high latitude $\mathrm{CHs}$ are higher than that of photospheric plasma.

Active Nests and Active Longitudes: Takahashi (1993) and Kozuka et al. (1993) have noted the X-ray counterparts of active longitudes and nests (Zwaan 1987) seen in other ways. It is seen from Figure 5 that there are fundamentally two active longitudes, around $0^{\circ}$ and $180^{\circ}$ in the Yohkoh time interval. The $\delta$-type sunspots producing many flares often appear in active longitudes. It is interesting to note here that these active longitudes correspond to giant bipolar magnetic regions (GBMR) situated on the global neutral line (Saito et al. 1993). On the basis of the 'triple dipole model' (Saito et al. 1989) of global solar magnetic field distribution, Saito predicted the existence of very large coronal loop structures above GBMRs in active longitudes. Such very large coronal loop structures have actually been observed on the limb in FFI images when the GBMR crosses the limb (Saito et al. 1993).

Anemone-ARs: The Yohkoh/SXT has revealed that many of ARs appearing in coronal holes show structure similar to "sea-anemone" as described above. Such ARs are called anemone-ARs (see Figure 4). The anemone-ARs tend to appear in coronal holes (far from the active longitudes) in the northern hemisphere during January 1992 - March 1993 (Shibata et al. 1993c). This tendency is opposite to the number of sunspots, which are more in the southern hemisphere during the same period. This as well as the apparently less-sheared field structure of the anemone-ARs suggest that the anemone-ARs are a signature of less-active ARs (i.e., $\alpha$-type spots) in spite of much magnetic flux, and that the origin of activity (or anti-activity) may be deeply rooted in the convection zone since the origin of the anemone-ARs seems to be closely related to the origin of coronal holes.

Topology of Global Coronal Magnetic Fields: The SXT movie has clearly shown that the topological pattern of global coronal magnetic fields changes with time as ARs emerge (as discussed above), evolve, and decay. It is not a rare phenomenon that the magnetic 


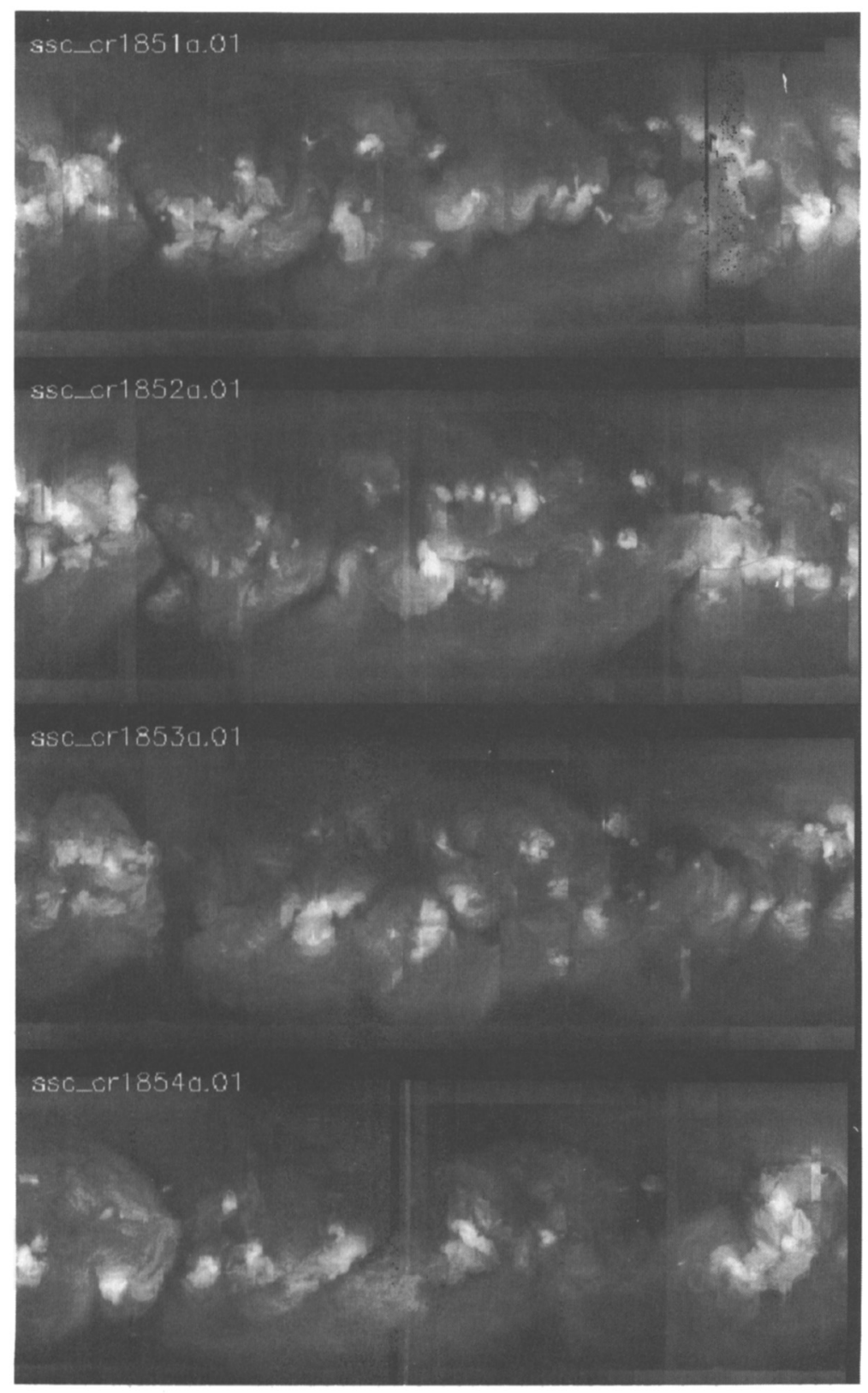

Figure 5. X-ray synoptic charts during Carrington rotation 1851-1854 (Jan. - Apr. 1992) made by Slater \& Lemen (1993).

field in an $A R$ in the northern hemisphere connects to an $A R$ in the southern hemisphere (i.e., trans-equatorial loops), as observed by Svestka \& Howard (1979). The boundary of a coronal hole $(\mathrm{CH})$ depends strongly on the evolution of the neighboring AR. In some cases, a small $\mathrm{CH}$ appeared after the filament eruption (Watanabe et al. 1992). It is also found that the shape of loops in the ARs in active longitudes are often sheared and twisted; the direction of the shear is opposite in northern and sourthen hemispheres, and 


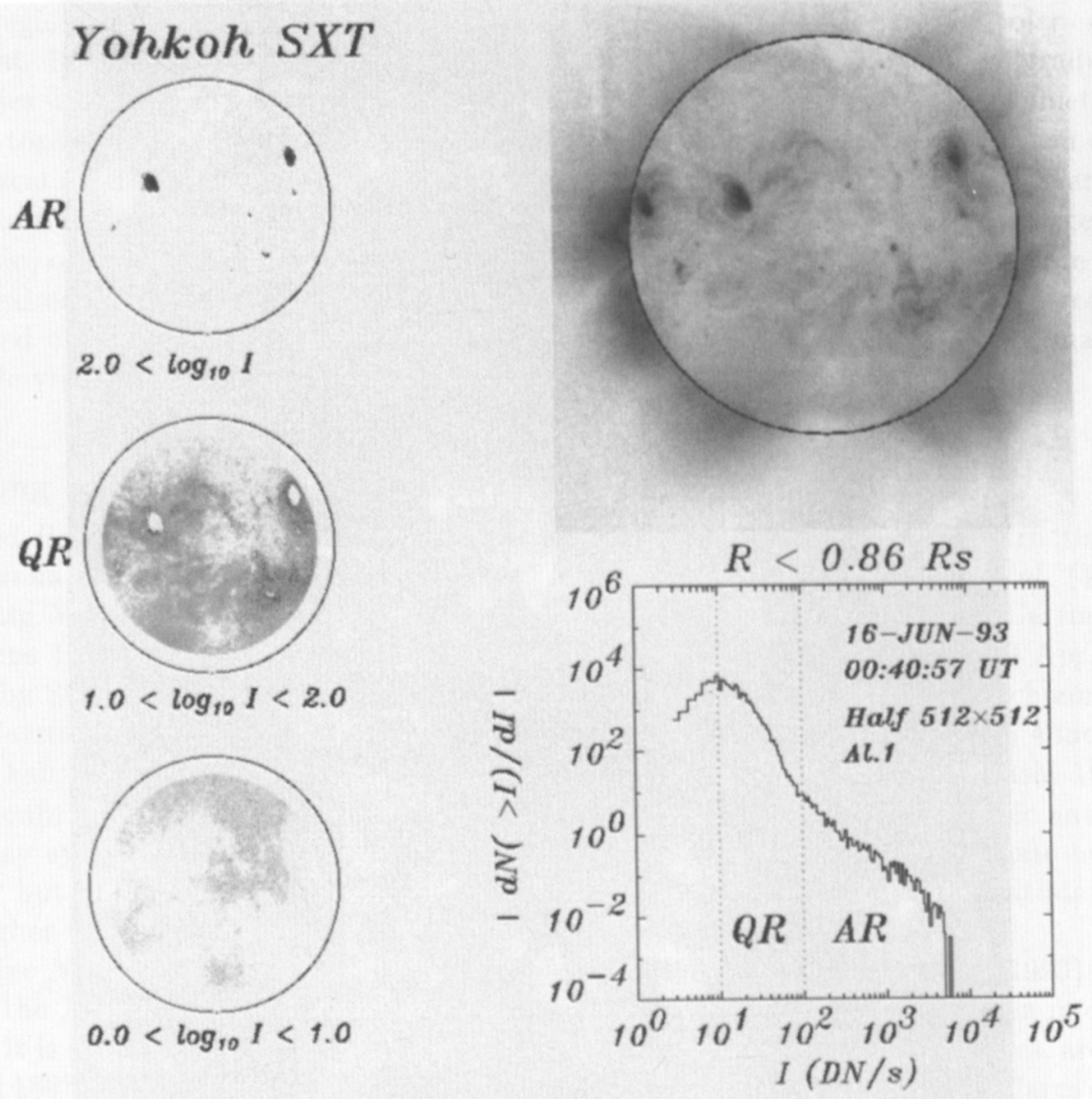

FIGURE 6. The number-distribution of soft X-ray intensity per pixel in an SXT/FFI image taken with thin aluminum filter on 1993 June 16 (shown in right bottom), i.e., the number of pixels with intensity between I and I $+\mathrm{dI}$ as a function of I. The FFI image is shown in the right top, and the three images in the left show the spatial distributions of AR-pixels (top), QR-pixels (middle), and darker pixels (including coronal hole). The pixels near the limb and outside the Sun are excluded from statistics. All these figures are from Hara (1993).

is consistent with the direction expected from the Coriolis force acting on a rising (and expanding) magnetic flux tube in the convection zone. This suggests that not only the formation of ARs but also the generation of the twist and shear (i.e., origin of activity) might be deeply rooted in the convection zone.

Long-Term Variation of the Soft X-ray Intensity: According to Hara (1993), the fraction of total soft X-ray intensity (observed by SXT) emitted from ARs is about 90 percent in an active phase (e.g., in Oct. 1991), while it is about 30 percent in a less active phase (e.g., in June, 1992). Hara (1993) further studied the distribution of soft X-ray intensity per pixel in SXT/FFI images (Figure 6), and found that the distribution pattern is different between quiet regions (QR) and active regions (AR). Figure 6 shows that the distribution for AR-pixels is nearly power-law while that for QR-pixels is not. This power-law distribution for AR-pixels reminds us of the power-law distribution of the 
solar flare occurrence against total flare energy (e.g., see Hudson 1991) and the idea of episodic heating (Sturrock et al. 1984; Parker 1988). Hara (1993)'s result suggests that the different heating mechanisms might work in $\mathrm{QR}$ and $\mathrm{AR}$.

\section{Summary}

The Yohkoh/SXT has revealed that the solar corona is much more dynamic than has been thought, i.e., the non-flaring corona is full of loop brightenings, jets, AR expansions from continual growth of EFRs to transient CMEs, global restructuring of coronal magnetic field beyond AR size, and so on. These are controlled by global pattern of magnetic field, such as coronal holes, active longitudes, and active nests. The total X-ray intensity is dominated by ARs (especially in active longitudes) in active phase, but the detailed spectral characteristics of X-ray emission seem to depend on the evolutionary phase of ARs.

Acknowledgments. The video movie shown during the conference was made with the help of the following people; L. Acton, H. Ando, M. Bruner, S. Freeland, J. Hirayama, H. Hudson, J. Khan, J. Lemen, G. Linford, R. Matsumoto, M. Morrison, N. Nitta, Y. Ogawara, G. Slater, K. T. Strong, S. Tsuneta, and Y. Uchida. I would like to thank these people for their excellent work. I would also like to sincerely thank Dr. H. S. Hudson for his many helpful comments on this manuscript, and Prof. T. Hirayama for encouragement and useful discussion. Thanks are also due to Y. Ishido, H. Hara, G. Kawai, T. Takahashi, Y. Kozuka, and Prof. T. Saito for allowing me to use their results before publication in the conference talk and/or in this manuscript. Finally, I greatly acknowledge all Yohkoh team members for their tremendous contribution on developing both hardware and software, and on doing the daily work of operating Yohkoh successfully.

\section{REFERENCES}

Acton, L., Tsuneta, S., Ogawara, Y., Bentley, R., Bruner, M., Canfield, R., Culhane, L., Doschek, G., Hiei, E., Hirayama, T., Hudson, H., Kosugi, T., Lang, J., Lemen, J., Nishimura, J., Makishima, K., Uchida, Y. \& Watanabe, T. 1992 The Yohkoh Mission for high-energy solar physics. Science 258, 618-625.

ANToNUCCI, E. 1994 Variations of coronal radiations in X-ray related to coronal holes, active region loop systems, bright points. In The Sun as a Variable Star: Solar and Stellar Irradiance Variations (ed. J.M. Pap, C. Fröhlich, H.S. Hudson \& S.K. Solanki). Cambridge University Press, in press.

CHou, D. Y. 1993 Structure of emerging flux regions. In The Magnetic and Velocity Fields of Solar Active Regions (ed. H. Zirin, G. Ai \& H. Wang). Astronomical Society of the Pacific Conference Series Vol. 46, pp. 471-478.

Hanaoka, Y. Kurokawa, H., Enome, S., Nakajima, H., Shibasaki, K., Nishio, M., Takano, T., Toril, C., Sekiguchi, H., Kawashima, S., Bushimata, T., Shinohara, N., Irimajiri, Y., Koshishi, H., Shiomi, Y., Nakai, Y., Funakoshi, Y., Kitai, R., IshIURA, K. \& KimURA, G. 1993 Simultaneous observations of a prominence eruption followd by a coronal arcade formation in radio, soft X-rays and $\mathrm{H} \alpha$. Publ. Astron. Soc. Japan, in press.

Hara, H., Tsuneta, S., Lemen, J. R., Acton, L. W. \& McTiernan, J. M. 1992 Hightemperature plasmas in active regions observed with the Soft X-ray Telescope aboard Yohkoh. Publ. Astr. Soc. Japan 44, L135-L140.

HARA, H. 1993 The X-ray intensity distribution of the corona and its variability associated with solar activity. In New Look at the Sun with Emphasis on Advanced Observations of Coronal 
Dynamics and Flares - What Do We See with YOHKOH and Nobeyama Radioheliograph -. Univ. Academy Press, in press.

HaRvey, K. L. \& Martin, S. F. 1973 Ephemeral active regions. Solar Phys. 32, 389-402.

Harvey, K. L., NitTa, S., Strong, K. T. \& Tsuneta, S. 1993 The relationship of X-ray bright points to the photospheric magnetic fields. In $X$-ray Solar Physics from Yohkoh (ed. Y. Uchida, H. Hudson, T. Watanabe \& K. Shibata). Univ. Academy Press, in press.

HAYASHI, K. 1992 Rotation of coronal holes. Undergraduate thesis (in Japanese), Dept. Astronomy, Univ. Tokyo.

Hiei, E., Hundhausen, A. J. \& Sime, D. G. 1993 Reformation of a coronal helmet streamer by magnetic reconnection after a coronal mass ejection. J. Geophys. Res., in press.

Hudson, H. S. 1991 Solar flares, microflares, nanoflares, and coronal heating. Solar Phys. 133, 357-369.

Hudson, H. S. 1994 Spatial resolution of solar total irradiance variability: The Yohkoh whitelight observations. In The Sun as a Variable Star: Solar and Stellar Irradiance Variations (ed. J.M. Pap, C. Fröhlich, H.S. Hudson \& S.K. Solanki). Cambridge University Press, in press.

Hudson, H. S. 1993 The Yohkoh context for high-energy particles in solar flares. In Proc. Waterville Workshop on Solar Flares, in press.

Ishido, Y., Shibata, K., TANAKa, N. \& KURoKaWA, H. 1992 Observation of EFRs using SXT. In Proc. of "Study of Solar Active Phenomena induced by Magnetic Shear - New Results of Yohkoh Observations -" held in Mitaka, Tokyo, Japan at Feb. $12-14$ in 1992, pp. 145-149.

Kawai, G., Kurokawa, H., Tsuneta, S., Shimizu, T., Shibata, K., Acton, L. W., Strong, K. T. \& NiTTA, N. 1992 Comparison between $\mathrm{H} \alpha$ and Yohkoh soft X-ray images of emerging flux regions. Publ. Astr. Soc. Japan, 44, L193-L198.

KAWAI, G. 1993 Emerging flux regions observed in soft X-ray and H-alpha. In New Look at the Sun with Emphasis on Advanced Observations of Coronal Dynamics and Flares - What Do We See with YOHKOH and Nobeyama Radioheliograph -, in press.

Klimchuk, J. A., Acton, L. W., Harvey, K. L., Hudson, H. S., Kluge, K. L., Sime, D. G., Strong, K. T. \&. Watanabe, Ta. 1993 Coronal eruptions observed by Yohkoh. In $X$-ray Solar Physics from Yohkoh (ed. Y. Uchida, H. Hudson, T. Watanabe \& K. Shibata). Univ. Academy Press, in press.

Kozuka, Y., Watanabe, Ta., Kojima, M., Ohyama, M., Tsuneta, S., Saito, T. \& Watari, S. 1993 Large scale coronal and solar wind structures. In $X$-ray Solar Physics from Yohkoh (ed. Y. Uchida, H. Hudson, T. Watanabe \& K. Shibata). Univ. Academy Press, in press.

Matsumoto, R., Tajima, T., Shibata, K. \& KaIsig, M. 1993 Three-dimensional magnetohydrodynamics of the emerging magnetic flux in the solar atmosphere. Astrophys. J. 414, 357-371.

Mcallister, A., Uchida, Y., Tsuneta, S., Strong, K. T., Acton, L. W., Hiei, E., Bruner, M. E., Watanabe, TA. \& Shibata, K. 1992 The structure of the coronal soft X-ray source associated with the dark filament disappearance of 1991 September 28 using the Yohkoh Soft X-ray Telescope. Publ. Astr. Soc. Japan 44, L205-L210.

Nitta, N., Bastian, T. S., Aschwanden, M. J., Harvey, K. L. \& Strong, K. T. 1992 Simultaneous observations of coronal bright points in $\mathrm{X}$-ray and radio wavelengths. $\mathrm{Publ}$. Astr. Soc. Japan 44, L167-L172.

Ogawara, Y., Takano, T., Kato, T., Kosugi, T., Tsuneta, S., Watanabe, T., Kondo, I. \& UchidA, Y. 1991 The Solar-A Mission: An overview. Solar Phys. 136, 1-16.

Ogawara, Y., Acton, L. W., Bentley, R. D., Bruner, M. E., Culhane, J. L., Hiei, E., Hirayama, T., Hudson, H. S., Kosugi, T., Lemen, J. R., Strong, K. T., Tsuneta, S., Uchida, Y., Watanabe, T. \& Yoshimori, M. 1992 The status of Yohkoh in orbit: An introduction to the initial scientific results. Publ. Astr. Soc. Japan 44, L41-L44.

PARKer, E. N. 1988 Nanoflares and the solar X-ray corona. Astrophys. J. 330, 474-479.

Rosner, R., Tucker, W. H. \& VAIANA, G. S. 1978 Dynamics of the quiescent solar corona. Astrophys. J. 220, 643-665. 
Saito, T., Ohкi, T., Olmsted, C. \& AKasofu, S. I. 1989 A representation of the magnetic neutral line on the solar source surface in terms of the Sun's axial dipole at the center and two equatorial dipoles in the photosphere. J. Geophys. Res. 94, 14993-14999.

Saito, T., Minami, S., Kozuka, Y., Takahashi, T., Hudson, H., Tsuneta, S. \& WatanABE, T. 1993 Large-scale structure of the solar plasma corona - An analysis of it Yohkoh SXT images -. In Proc. of the Fourth International Conference on Plasma Physics and Controlled Nuclear Fusion (ESA SP-351). pp. 215-218.

Shimizu, T., Tsuneta, S., Acton, L. W., Lemen, J. R. \& Uchida, Y. 1992 Transient brightenings in active regions observed by the Soft X-ray Telescope on Yohkoh. Publ. Astr. Soc. Japan 44, L147-L153.

Shibata, K., Tajima, T., Steinolfson. R. \& Matsumoto, R. 1989 Two-dimensional magnetohydrodynamic model of emerging magnetic flux in the solar atmosphere. Astrophys. $J$. 345, 584-596.

Shibata, K. 1991 Theoretical models of solar flares. In Flare Physics in Solar Activity Maximum 22 (ed. Y. Uchida, R. Canfield, T. Watanabe \& E. Hiei). Lecture Note in Physics No. 387, Springer Verlag, pp. 205-218.

Shibata, K., Nozawa, S. \& Matsumoto, R. 1992a Magnetic reconnection associated with emerging magnetic flux. Publ. Astr. Soc. Japan 44, 265-272.

Shibata, K., Ishido, Y., Acton, L. W., Strong, K. T., Hirayama, T., Uchida, Y., McAllister, A. H., Matsumoto, R., Tsuneta, S., Shimizu, T., Hara, H., Sakurai, T., Ichimoto, K., Nishino, Y. \& Ogawara, Y. 1992b Observation of X-ray jets with the Yohkoh Soft X-ray Telescope. Publ. Astr. Soc. Japan 44, L173-L179.

Shibata, K., Nitta, N., Matsumoto, R., Tajima, T., Yokoyama, T., Hirayama, T. \& HUDSON, H. 1993a Two types of interaction between emerging flux and coronal magnetic field. In X-ray Solar Physics from Yohkoh (ed. Y. Uchida, H. Hudson, T. Watanabe \& K. Shibata). Univ. Academy Press, in press.

Shibata, K., Nitta, N., Strong, K. T., Matsumoto, R., Yokoyama, T., Hirayama, T., HUdSon, H. \& OGaWARA, Y. 1993b A gigantic coronal jet ejected from a compact active region in a coronal hole. Nature, submitted.

Shibata, K., NitTa, N. \& Hara, H. 1993c Characteristics of active regions appearing in coronal holes, to be submitted.

SLATER, G. \& LEMEN, J. 1993, private communication.

Strong, K. T., Harvey, K. L., Hirayama, T., Nitta, N., Shimizu, T. \& Tsuneta, S. 1992 Observations of the variability of coronal bright points by the Soft X-ray telescope on Yohkoh. Publ. Astr. Soc. Japan 44, L161-L166.

Sturrock, P. A., Kaufman, P., Moore, R. L. \& SMith, D. F. 1984 Energy release in solar flares. Astrophys. J. 94, 341-357.

StURrock, P. A. 1992 Emerging picture of eruptive solar flares. In Proc. IAU Colloq. No. 133, "Eruptive Solar Flares" (ed. Svestka, Z., Jackson, B. L. \& Machado, M. E. ). Spring-Verlag, pp. 397-409.

Su, Q., Hirayama, T., Shibata, K., Ichimoto, K., Tsuneta, S., Hara, T., Acton, L. \& LEMEN, J. 1993 Soft X-ray loops at the beginning of a rapidly growing sunspot group, in preparation.

SVESTKA, Z. \& HowARD, R. 1979 Interconnecting loops: morphology of the sudden brightenings. Solar Phys. 63, 297-318.

TAKAHASHI, T. 1993 Long-term variation of large scale solar X-ray structures with the Soft X-ray Telescope aboard Yohkoh satellite. Master thesis (in Japanese), Dept. of Astronomy, Univ. of Tokyo.

Timothy, A. F., Krieger, A. S. \& Vaiana, G. S. 1975 The structure and evolution of coronal holes. Solar Phys. 42, 135-156.

Tsuneta, S., Acton, L. W., Bruner, M., Lemen, J., Brown, W., Caravalho, R., Catura, R., Freeland, S., Jurcevich, B., Morrison, M., Ogawara, Y., Hirayama, T. \& Owens, J. 1991 The Soft X-ray Telescope for the Solar A Mission. Solar Phys. 136, 37-67. 
Tsuneta, S., Takahashi, T., Acton, L. W., Bruner, M. E., Harvey, K. L. \& Ogawara, Y. 1992 Global restructuring of the coronal magnetic fields observed with the Yohkoh Soft X-ray Telescope. Publ. Astr. Soc. Japan 44, L211-L214.

Tsuneta, S., Hara, H., Shimizu, T., Acton, L. W., Strong, K. T., Hudson, H. \& OgaWARA, Y. 1992 Observation of solar flare at the limb with the Yohkoh Soft X-ray Telescope. Publ. Astr. Soc. Japan 44, L63-L69.

Tsuneta, S. \& LEmen, J. R. 1993 Dynamics of the solar corona observed with the Yohkoh Soft X-ray Telescope. In Proc. "Advances in Stellar and Solar Coronal Physics" (ed. J. Linsky \& S. Serio). Kluwer, in press.

Tsuneta, S. 1993 Highlights of Yohkoh results. In Proc. of the Fourth International Conference on Plasma Physics and Controlled Nuclear Fusion (ESA SP-351). pp. 75-83.

Uchida, Y., Mcallister, A., Strong, K. T., Ogawara, Y., Shimizu, T., Matsumoto, R. \& HUdSON, H. 1992 Continual expansion of the active-region corona observed by the Soft X-ray Telescope on Yohkoh. Publ. Astr. Soc. Japan 44, L155-L160.

UCHIDA, Y. 1993 Some initial results from the Yohkoh observations of solar high-temperature and high-energy phenomena. In Proc. of COSPAR Symp., in press.

Watanabe, Ta., KozUka, Y., Ohyama, M., Kojima, M., Yamaguchi, K., Watari, S., Tsuneta, S., Joselyn, J. A., Harvey, K. L., ACton, L. W. \& Klimchuk, J. A. 1992 Coronal/interplanetary disturbances associated with disappearing solar filaments. Publ. Astr. Soc. Japan 44, L199-L204.

WEBB, D. F . 1981 Active region structures in the transition region and corona. In Proc. Skylab Workshop "Solar Active Region" (ed. F. Q. Orrall). U. Colorado Press, pp. 165-198.

YoKoyama, T. \& ShIBATA, K. 1993 Numerical simulation of magnetic reconnection associated with emerging flux in the solar atmosphere. In Proc. of the Fourth International Conference on Plasma Physics and Controlled Nuclear Fusion (ESA SP-351). pp. 203-206.

ZWAAN, C. 1987 Elements and patterns in the solar magnetic field. Ann. Rev. Astr. Ap. 25, 83-111. 